\title{
e-Phaïstos
}

e-Phaïstos Revue d'histoire des techniques / Journal of the history of technology

VIII-2 | 2020

Aviation : émergence d'un complexe technique, 1900-1930

\section{Concevoir et fabriquer des avions dans l'entre- deux-guerres en France}

Designing and Manufacturing Aircrafts in the Inter-War Period in France

\section{David Culpepper}

\section{OpenEdition}

Journals

Édition électronique

URL : https://journals.openedition.org/ephaistos/8142

DOI : 10.4000/ephaistos.8142

ISSN : 2552-0741

Éditeur

IHMC - Institut d'histoire moderne et contemporaine (UMR 8066)

Référence électronique

David Culpepper, «Concevoir et fabriquer des avions dans l'entre-deux-guerres en France », e-Phaïstos [En ligne], VIII-2 | 2020, mis en ligne le 27 octobre 2020, consulté le 16 septembre 2021. URL : http:// journals.openedition.org/ephaistos/8142 ; DOI : https://doi.org/10.4000/ephaistos.8142

Ce document a été généré automatiquement le 16 septembre 2021.

Tous droits réservés 


\title{
Concevoir et fabriquer des avions dans l'entre-deux-guerres en France
}

\author{
Designing and Manufacturing Aircrafts in the Inter-War Period in France
}

David Culpepper

1 Au début de l'aviation, la fiabilité était moins importante que le poids, les premiers essais n'ayant comme but que de décoller ${ }^{1}$. Mais avec le temps et le progrès technologique qui l'accompagnait, d'autres facteurs sont devenus plus importants, comme la sécurité. Après l'établissement de la sécurité du vol régulier et l'introduction des avions en tant que machines de guerre, les performances au combat sont devenues essentielles et même prioritaires. L'avion militaire était à la fois un produit de l'industrie (partiellement conçu en fonction des coûts et de la facilité de production) et un appareil de guerre (conçu pour la performance) ${ }^{2}$. Ces deux aspects de l'avion militaire étaient, pourtant, diamétralement opposés. Un avion de guerre en service paraissait donc être le résultat d'un compromis entre les divers facteurs de performance et de conditions de production ${ }^{3}$. C'était alors à l'ingénieur d'arbitrer entre ces besoins divergents relatifs à la conception et la production.

2 Selon Alexander de Seversky, le célèbre inventeur et stratège de l'aviation, les caractéristiques d'un bon avion de guerre étaient :

\footnotetext{
«la vitesse, la manœuvrabilité, et l'assurance d'une alimentation adéquate. Elle veut dire la facilité de contrôle aux moments critiques [...]; toute la protection possible pour le pilote [...]; un avion construit avec le compromis entre le besoin d'un instrument de défense d'une efficience élevée et le besoin d'une machine aérodynamique à la perfection ${ }^{4}$ ».
}

D'autres facteurs se dessinaient à travers la nature de l'armement, la visibilité et l'installation de nouvelles technologies, comme les dispositifs de communication. Ils prenaient de la place dans un espace déjà contraint et étriqué5. Il existait donc plusieurs critères de bonne performance pour un avion, sans que, pour autant, toutes les qualités d'un aéroplane puissent être réunis dans un même équipement. Il revenait 
donc à l'ingénieur de déterminer les caractéristiques de l'appareil, en fonction du rôle qui lui était dévolu. Si l'avion était conçu spécifiquement pour la production en série, il risquait de ne pas disposer des atouts nécessaires en termes de poids et d'aérodynamique. Il fallait donc concilier la quantité et la performance. S'il y avait trop peu d'avions, l'armée risquait d'être vite accablée par les forces ennemies. Pourtant, si les appareils étaient de qualité inférieure, il leur serait impossible de se confronter à des adversaires au matériel plus performant.

\section{Concevoir un avion de guerre}

4 L'idéal pour un chasseur était d'être léger et très réactif, le contraire étant vrai pour un bombardier $^{6}$. Les champs de recherches résidaient dans l'amélioration des profils aérodynamiques, le développement des matériels, des alliages, pour obtenir un meilleur rapport force/poids, une réduction de la masse des moteurs et l'amélioration en efficacité par la détermination du design structurel ${ }^{7}$. Le soudage, qui permettait d'obtenir des lignes aérodynamiques, présentait des difficultés de production et d'interchangeabilité ${ }^{8}$. Le soin apporté à la conception de chaque pièce d'un avion était rigoureux ; même les boulons étaient conçus spécialement pour offrir le poids le plus faible possible ${ }^{9}$. Une augmentation dans ce domaine, même insignifiante en apparence, pouvait détériorer significativement la vitesse ascensionnelle. L'alternative consistait à emporter moins de carburant et/ou de munitions, ce qui avait comme effet de réduire la distance franchissable et la longévité au combat ${ }^{10}$. Afin de construire des avions capables de poursuivre et d'abattre des bombardiers, le profil du chasseur devait le conduire à perdre en manœuvrabilité, au profit de la vitesse et du rayon d'action, par l'emport de davantage de réservoirs et d'armes plus lourdes ${ }^{11}$.

Fig.1. Vue en demi-coupe du chasseur monoplace Messerschmidt Bf 109

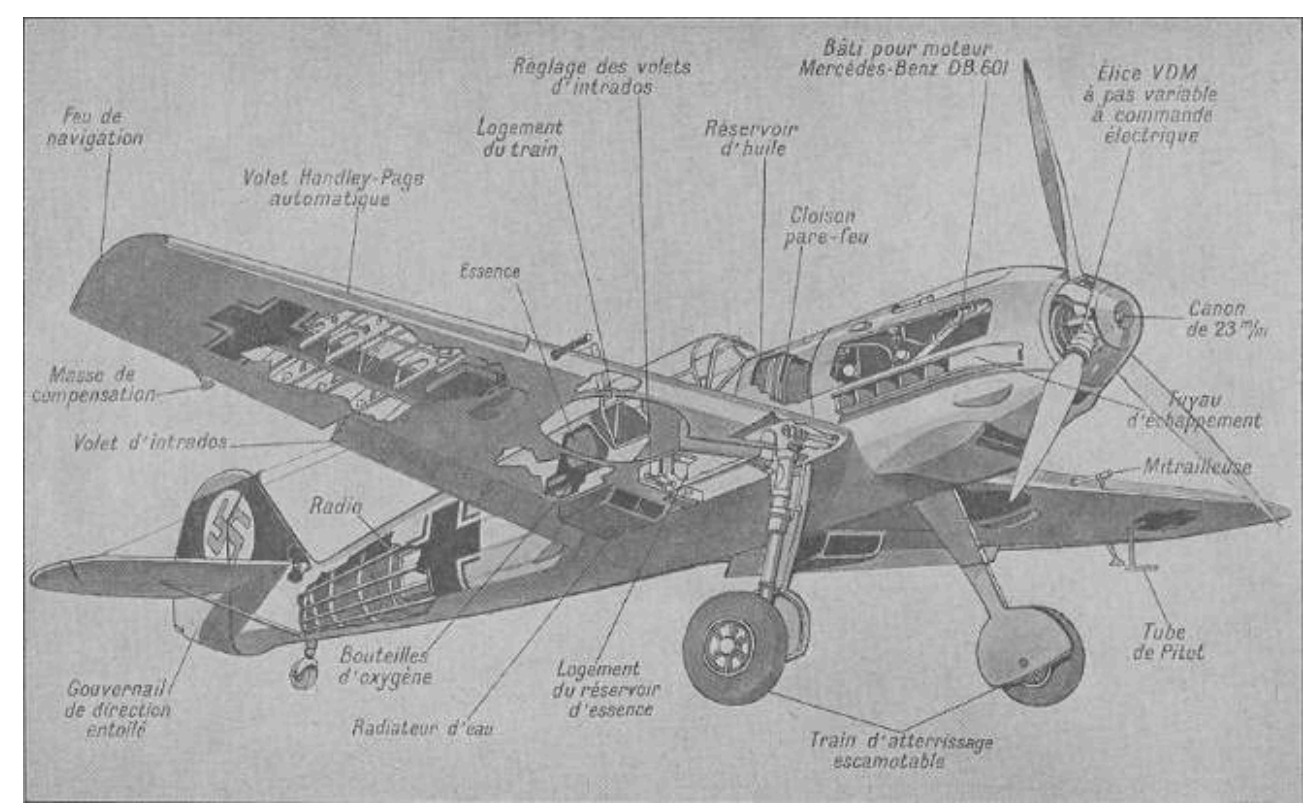

Cet avion de chasse est le plus répandu dans la Luftwaffe au début de la Seconde Guerre mondiale. Appareil le plus rapide au début du conflit, il se caractérise par ses qualités indéniables qui lui confèrent une supériorité sur la plupart de ses équivalents lors de la Campagne de France du printemps 1940, mais aussi par une conception qui limite les améliorations ultérieures.

Extrait de La science et la vie, avril 1940 
Les ingénieurs devaient constamment incorporer les nouvelles recherches et avancées scientifiques. Elles étaient nombreuses pendant l'entre-deux-guerres, période au cours de laquelle les constructeurs d'avions abandonnaient les matériaux en bois et toile en faveur de la généralisation des alliages ${ }^{12}$. La conception d'un avion était plus complexe. Elle englobait davantage que le design de l'appareil lui-même. Il s'agissait d'une tâche incroyablement compliquée, associant beaucoup de facteurs à considérer. L'ingénieur devait concevoir les outils à utiliser, l'organisation de la production, les composants à fabriquer, leurs matières et le processus de fabrication à travers la chaîne de montage et d'assemblage de l'avion. Les entreprises aéronautiques ont dû prendre en compte les avancées techniques prévisibles entre le commencement du développement et la mise en service de l'avion ainsi que les exigences changeantes de la guerre. De plus, il fallait que tout soit prêt en même temps au sein des firmes pour que la production commence le plus vite possible. L'ingénieur en aéronautique britannique Frederick George Miles l'a rappelé en ces termes: "Il n'a existé aucune certitude de conception jusqu'au moment où le premier avion de production a volé $»^{13}$.

Deux façons de concevoir un avion performant pouvaient être utilisées avec la modification d'une forme existante ou la création d'une forme nouvelle. La première solution passait par l'utilisation de matériaux nouveaux, supérieurs, comme des alliages légers, l'emploi de nouvelles techniques d'ingénierie pour réduire le poids et l'incorporation d'améliorations aérodynamiques ${ }^{14}$. Les nouveaux avions, en raison de leur complexité, étaient toujours confrontés à des problèmes imprévus. Il était donc plus efficient de construire des appareils déjà éprouvés et, pour la production, de se concentrer sur quelques modèles possédant des variantes correspondant à des missions spécifiques ou à des améliorations. Pendant la Seconde Guerre mondiale, la plupart des avions construits aux États-Unis étaient déjà conçus, ce qui permit de sauter plusieurs étapes de développement des prototypes et de gagner ainsi un à deux ans (Simonson 1960 : 375). De l'autre côté de la Manche, le célèbre Spitfire britannique fut construit en 20000 exemplaires, mais avec pas moins de vingt-quatre modèles différents (Agnew 1993 : 126). 
Fig.2. Vue en demi-coupe du chasseur monoplace Supermarine « Spitfire »

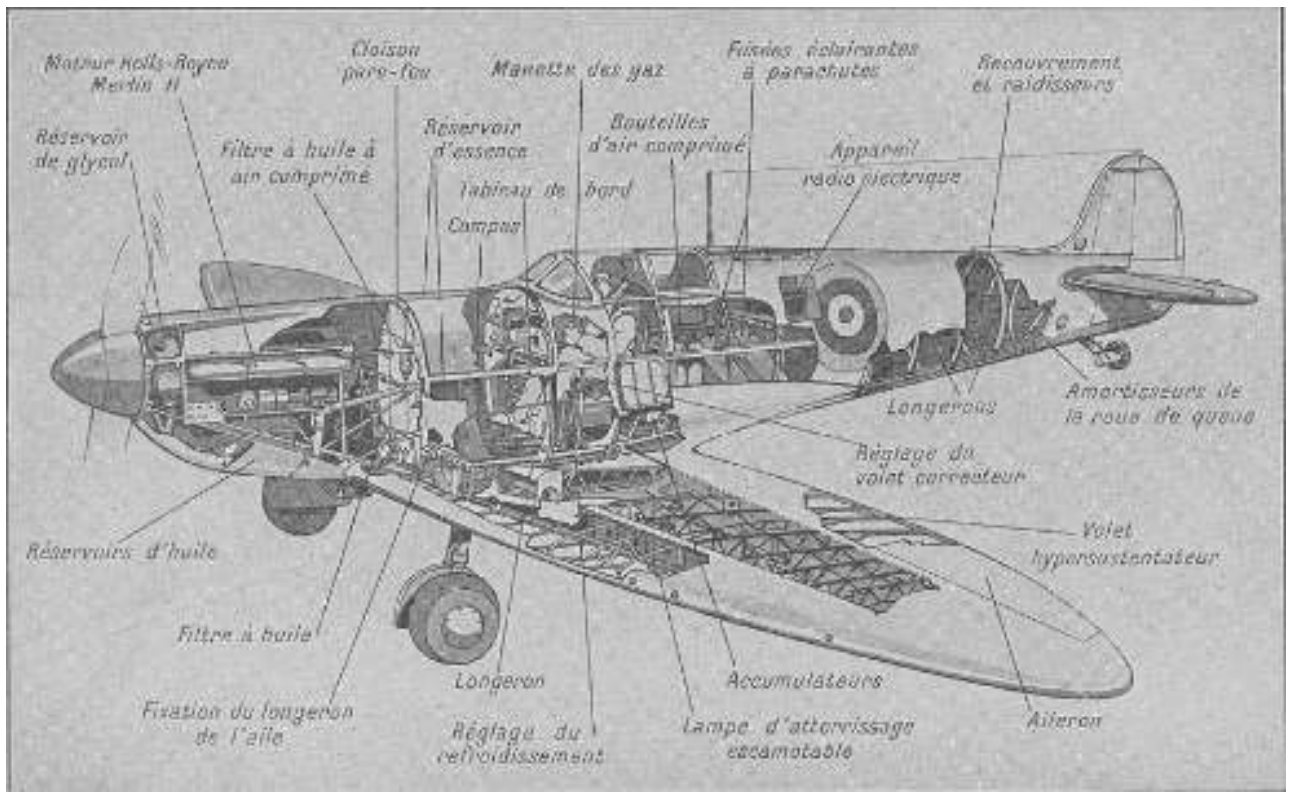

Ce dessin présente les principaux organes du Spitfire, célèbre avion de chasse britannique, qui entre en service quelques mois avant la Seconde Guerre mondiale. II se caractérise par la puissance de son moteur Rolls Royce et par un armement logé dans les ailes de l'appareil. II se distingue par ses

qualités aérodynamiques. Construits en alliage léger, certains des premiers modèles reçoivent encore une hélice en bois bipale.

Extrait de La science et la vie, avril 1940

7 La France opta malgré tout pour la seconde voie, celle de multiplier les modèles, les chefs militaires n'étant pas satisfaits (à raison) des avions existants. En 1940, l'armée de l'air alignait vingt-six modèles différents d'avions tandis que la Luftwaffe n'en avait que six (Garraud 2002: 51). Celle-ci ne possédait qu'un seul chasseur monoplace et un chasseur bimoteur en service en 1940, tandis que l'Armée de l'air disposait de six types de chasseurs (Garraud 2001 : 11). Il n'existait pas vraiment de solutions pour accélérer la mise en production d'avions en grandes séries, hormis l'organisation d'un dispositif rigoureux d'approvisionnements en matières premières et composants, des usines bien équipées en machines-outils et des ouvriers formés à l'utilisation de ces machines. Afin de gagner du temps, la planification de la production et la commande des matériels pouvaient être effectuées de concert avec le développement de l'avion. Pourtant, de tels choix finissaient parfois par entraver la production et déboucher sur un gaspillage des ressources, suite à des changements dans la conception de l'avion ${ }^{15}$. Afin d'assurer une production rapide, l'ingénieur pouvait tracer un avion moins aérodynamique, mais associant moins de pièces différentes, actions qui auraient un impact sur le poids et la performance de la machine ${ }^{16}$. Plus tard dans la guerre, les tensions sur les approvisionnements ont poussé les Allemands à assembler des avions présentant beaucoup de pièces préexistantes, ce qui a eu comme effet d'aligner des avions confrontés à une multitude de problèmes techniques (Zeitlin 1995: 65). Avant de commencer la production, il était préférable de s'appuyer sur un design stable ${ }^{17}$. Cela signifiait que l'avion pouvait recevoir de petites modifications en cours de production, mais sans remise en cause de ses principales caractéristiques structurelles. À ce propos, le contrôleur français Chossat déclara que : 
«Trop souvent les avions modernes n'étaient pas complètement définis au moment où était lancée la fabrication de série. Un avion est défini lorsque tous les détails de construction et d'aménagement sont précisés, de façon que les liasses de dessins permettant la fabrication de la série puissent être définitivement arrêtées ${ }^{18} »$.

\section{Vers la mise en production}

8 La première étape au terme de la conception de l'avion correspond à la planification des processus de fabrication et d'assemblage. Il fallait penser la production de chaque composant : l'outillage et l'approvisionnement nécessaires, sa forme, l'ordre des opérations et les outils. La cadence de production de la pièce devait être précisément déterminée pour considérer les moyens nécessaires et les conditions de production. La même logique prévalait pour l'ensemble de l'appareil: il fallait décider comment diviser les ensembles et les sous-ensembles, leur ordre, les outils et la capacité de production désirée ${ }^{19}$. Des problèmes de production étaient soulevés par la complexité de l'assemblage des pièces et de leur forme. De telles contraintes étaient plus facilement maîtrisées dans un atelier que sur une chaîne de montage. Dans le premier cas, les ouvriers étaient habitués à faire des modifications et à s'adapter aux spécificités de chaque aspect de la fabrication et de l'assemblage ${ }^{20}$.

Dans les ateliers, une relation de confiance pouvait exister entre ouvriers et ingénieurs qui pouvaient communiquer entre eux. Mais avec la production en grande série, les conditions de production ont dû être adaptées. La qualification de la main-d'œuvre n'avait pas à être aussi poussée car la construction des appareils reposait sur des principes simplifiés, associés à une uniformisation des procédés ${ }^{21}$. Chaque pièce a été conçue afin d'obtenir une performance optimale, sans usure, dégradation, corrosion voire destruction. L'ingénieur était confronté à de nombreuses sortes d'acier, mais aussi d'autres métaux et alliages ${ }^{22}$. Des aciers spéciaux ont été employés pour les cylindres, vilebrequins. Les alliages à haute teneur en chrome et nickel apparus pendant la Première Guerre mondiale ont aussi été utilisés pour les soupapes d'échappement. D'autres aciers présentaient des nuances infinies, avec une composition comprenant une dose variable de chrome, magnésium et nickel ${ }^{23}$. Un inspecteur français déclara à ce propos que :

\footnotetext{
«Chaque pièce, PC (pièce composée) ou sous-ensemble, correspond à une définition géométrique prévue par un plan qui définit aussi la matière, l'état de la matière, le traitement, la protection de surface. En plus, un numéro d'ordre permet de situer la date et le lieu de fabrication » (Lucas $1988:$ 117).
}

Comme nous l'avons signalé, la conception d'un avion devait prendre en compte chaque pièce de l'avion, y compris le groupe motopropulseur. Certains moteurs étaient développés sur de longues périodes, afin d'améliorer leur robustesse, leur fiabilité et leur efficacité. Les designers d'avions étaient confrontés au choix du moteur. Ils ont dû concevoir leurs avions à partir des modèles de moteurs disponibles. Cette tendance a pu s'inverser. À la fin de l'entre-deux-guerres, en raison des exigences de vitesse et de puissance, les constructeurs de moteurs commençaient à produire des moteurs en 
fonction des performances et des spécifications des avions, ce qui laissait plus de choix aux ingénieurs ${ }^{24}$. En raison de la nature spécifique de leur fonctionnement, les paramètres de design des pales d'hélices étaient très contraignants. Il fallait considérer les charges aérodynamiques et centrifuges, la haute vitesse des tours de l'hélice, ainsi que la rigidité, la solidité et la forme très spécifique de ces pales ${ }^{25}$. La force centrifuge exercée sur une hélice était plus grande que les forces subies par le reste de l'avion ${ }^{26}$. De plus, des conflits survenaient entre la forme des pales et la finesse aérodynamique. Il fallait surtout que l'hélice échappe aux contraintes nées des vibrations ${ }^{27}$. Il n'était pas possible à l'époque de concevoir une hélice avec un rendement maximum à la vitesse exacte désirée. L'expérimentation de plusieurs types s'imposait pour parvenir au meilleur équilibre ${ }^{28}$. Selon Roy Fedden, célèbre ingénieur anglais, les concepteurs d'hélices avaient peut-être le travail le plus difficile ${ }^{29}$.

11 Tout interagissait dans une grande chaîne qui devait fonctionner sans heurt. Pourtant, avant de pouvoir évaluer la chaîne de montage, il fallait d'abord observer l'avion en action. Ce faisant, par retour d'expériences à la suite des campagnes d'essais, même les plans les plus méticuleux devaient être modifiés après observation des premiers moments de fonctionnement (Cohen 1991: 772). Les problèmes étaient plus aisés à dépasser quand la conception d'un appareil était intangible. Mais le progrès technique étant continu, il devait être incorporé pendant la production en série, notamment sous la pression des utilisateurs - les militaires - qui exigeaient des avions compétitifs. De petits progrès comme de grands changements intervenaient constamment, ce qui avait pour effet d'augmenter la complexité de la production et repoussait la mise en service des avions. Même après le lancement de l'assemblage, des modifications survenaient (qui nécessitaient des modifications post-production et/ou des évolutions du processus de production).

12 De plus, puisque les outils et les gabarits étaient spécifiquement conçus pour un avion donné, chaque changement dans la conception d'un appareil engendrait une adaptation des outils et des gabarits déjà adoptés, ce qui reportait ou contrariait les conditions de mise en production ${ }^{30}$. Par exemple, pour la construction du Bloch 155, une simple évolution du Bloch 152, il n'était pas possible d'employer les mêmes outils et gabarits ${ }^{31}$. Dans le cas des avions en cours fabrication, les reprises et modifications étaient réalisées avant leur livraison. Quant aux avions déjà acceptés ou même alignés par l'Armée de l'air, il fallait les renvoyer à l'usine pour qu'ils subissent les améliorations, ce qui avait pour effet, non seulement de retarder ou perturber leur utilisation, mais aussi la production en cours. En raison de la nature changeante de la guerre et des nouveaux besoins correspondants, un avion "moderne ", conçu cinq, six, ou même sept ans plus tôt, présentait des performances médiocres en comparaison de la technologie la plus récente. Il fallait donc faire des modifications afin d'arriver à la hauteur des nouvelles espérances militaires ou tout au moins pour rattraper une partie $\mathrm{du}$ retard sur les concurrents/ennemis. Les ailes du Dewoitine 520, un avion prometteur et déjà en production, ont dû être modifiées pour permettre, selon les exigences des chefs militaires, le passage du nombre de mitrailleuses de quatre à six. L'expérience acquise dès le début de la Drôle de guerre avait démontré la nécessité de disposer d'une plus grande puissance de feu ${ }^{32}$.

13 En parallèle, des problèmes dans la conception initiale, remarqués après la sortie de l'avion de l'usine, imposaient des modifications ultérieures. En plus des modifications exigées par la hiérarchie pour des raisons militaires, celles proposées par les pilotes 
d'escadrille pouvaient être prises en compte. Des changements étaient toujours engagés à partir de leur expérience. Souvent, des faiblesses et défauts ne se révélaient qu'après une période d'emploi étendue ${ }^{33}$. Pour le Bloch 151, par exemple, des problèmes de traînée mais aussi de refroidissement du moteur nécessitaient des modifications ultérieures considérables (Garraud 2002: 54). Le contrôleur Chossat affirma que "les utilisateurs constataient très fréquemment de multiples petites défectuosités techniques tenant aux conditions de montage ou de finition, et rendant les avions indisponibles pendant un certain temps $»^{34}$. À lui seul, le Dewoitine 520 subit plus que 100 modifications ; des 430 machines livrées à l'Armée de l'air, 150 (ou presque 35\%) étaient stockés dans des hangars au moment de l'armistice de 1940, en raison de modifications rendant caduque leur utilisation. ${ }^{35}$ Les contrôleurs Chossat et Sordes ont successivement décrit le processus ainsi :

« Tout matériel nouveau - et particulièrement lorsqu'il est délicat et compliqué comme un avion moderne - exige de nombreux mois d'adaptation, d'innombrables modifications, voire des transformations importantes, des aménagements et des renforcements, nécessitant, par suite, des périodes parfois très longues d'indisponibilités, avant qu'il soit en état de donner satisfaction aux utilisateurs. Il était donc facile de prévoir - ce que les faits ont malheureusement démontré - que les avions nouvellement livrés ou à sortir prochainement donneraient des déceptions et des déboires au cours d'une utilisation immédiate et intensive, et qu'il ne serait possible d'obtenir un plein rendement des unités qu'ils équiperaient, qu'au bout d'un temps relativement long ${ }^{36}{ }^{\prime \prime}$.

Et Sordes d'ajouter :

«L'histoire de chaque prototype n'est qu'une longue suite, pendant des semaines et des mois, d'essais successifs semés de difficultés sans cesse renaissantes et sans cesse vaincues. Car le nombre des inconnus est ici tel que le bureau d'études et le laboratoire sont impuissants à établir les tracés définitifs. Seul l'essai en vraie grandeur apporte la certitude avec bien entendu ce qu'il comporte de temps, de labeur et de dépense [...]. Ce prototype même mis au point de façon parfaite est encore susceptible de défaillances au cours de son utilisation en service, ce qui n'est pas surprenant quand nous voyons la clientèle de l'automobile, mécanisme infiniment plus simple cependant, faire sur la route la mise au point des premières séries. Il faudra donc le modifier pour porter remède aux défauts constatés et on voudra aussi le modifier pour y apporter des améliorations plus ou moins de détail ou le faire bénéficier de progrès nouveaux et il en résultera pour la production des perturbations profondes ${ }^{37} »$. 


\section{Concilier conception rapide et production de masse : le difficile apprentissage des constructeurs français}

15 Avant son application sur les avions, la production de masse était utilisée pour assembler les voitures et les wagons de marchandises, mais ceux-là étaient conçus pour une production simple et un coût unitaire faible plutôt que pour de hautes performances, comme cela était nécessaire avec un avion de guerre ${ }^{38}$. Pour un chasseur, les compromis en termes de performance et de mise en œuvre de la production pouvaient être réalisés jusqu'à un certain point seulement. Au moment de la conception d'un avion, celui-ci devait correspondre aux spécifications émises par l'armée. Pourtant, en période de réarmement, le design de l'avion devait répondre aux cahiers des charges, tout en faisant en sorte que l'appareil puisse être produit en masse. Avant l'avènement du réarmement, le besoin d'avoir des milliers d'exemplaires des avions ne s'imposait pas. En période de paix, les armées n'avaient pas besoin d'avions conçus pour une production intensive. La simplification des procédures de construction ainsi que des pièces ne paraissait pas nécessaires. Pendant cette période, la conception d'un appareil n'était pas contrariée par le processus de fabrication pour lequel aucune planification méticuleuse n'était nécessaire ${ }^{39}$.

16 Mais en France, y compris au début du réarmement, les prototypes n'étaient conçus qu'en fonction d'un souci de performance. La construction de ces avions était longue, "d'une complication inimaginable ", et réalisée de manière " artisanale ", à l'ancienne. Les petites séries de production exigeaient moins de réflexion préalable que la production de masse. Elles étaient pensées pour des ateliers d'ouvriers qualifiés associés à des outils diversifiés ${ }^{40}$. Dans ce cadre, après l'acceptation du prototype, il fallait que les ingénieurs refassent tous les dessins des éléments de l'appareil afin de les simplifier et rendre l'avion réalisable en grande série ${ }^{41}$. Le design initial d'un avion français devait en faire un appareil performant, qui répondait aux besoins spécifiés par l'armée. Ensuite seulement était envisagée une production plus simple, tout en conservant ses qualités initiales. Mais cette dissociation pouvait avoir des conséquences néfastes. L'avion fabriqué en série n'était plus le même que celui alors présenté à l'Armée de l'air. Des différences considérables entre la performance du prototype et celle des appareils produits apparaissaient. Elles mobilisaient des ressources financières, humaines, très importantes, associées à une importante perte de temps.

Le chasseur Bloch 150 est excellent exemple d'avion qui n'était pas dessiné pour être industrialisé. La conception a commencé en 1934, suite à une spécification technique fixée par le ministère de l'Air. Un an plus tard, la construction du prototype débute. Presque deux ans après la présentation de ses caractéristiques, une première tentative de vol est réalisée. Elle échoue ${ }^{42}$. L'avion est ensuite modifié et réussit à voler l'année suivante. Impressionnée par les premiers essais de la nouvelle version, l'armée de l'Air passe une commande importante. La production est lancée en mai 1938, mais il apparaît très vite que cet appareil est beaucoup trop compliqué à fabriquer en série. Il faut donc une reprise totale de la conception pour faire coïncider l'appareil avec une production intensive nécessaire pour amener l'aviation française au même niveau qu'en Allemagne.

18 Finalement, en avril 1939, l'armée de l'Air ne possédait encore que quatre Bloch 150, presque cinq ans après avoir déterminer ses besoins ${ }^{43}$. En plus du temps, de l'argent, du matériel, des outils et du personnel gaspillés, l'Armée de l'air reçoit un appareil qui est 
déjà dépassé, dans la mesure où il répond à des spécifications datant de 1934. En effet, à cette époque, les progrès aéronautiques étaient importants et rapides. Il était donc très difficile d'engager la production en série alors qu'elle n'avait pas été prise en compte au moment de la conception. Après cet échec, l'industrie aéronautique française s'est efforcée d'annoncer que ses nouveaux avions étaient conçus pour répondre à des besoins importants, afin de pouvoir rassurer la clientèle, notamment au moment de la diffusion des brochures promotionnelles et des présentations lors des salons ${ }^{44}$ et meetings aériens ${ }^{45}$. Ainsi, au Salon international de 1936, le prototype du Dewoitine 520 est exposé avec une campagne d'affiches qui indiquent qu'il a été conçu pour une production rapide et « en grande série ${ }^{46}$.

Fig 3. Le chasseur monoplace Dewoitine D.520

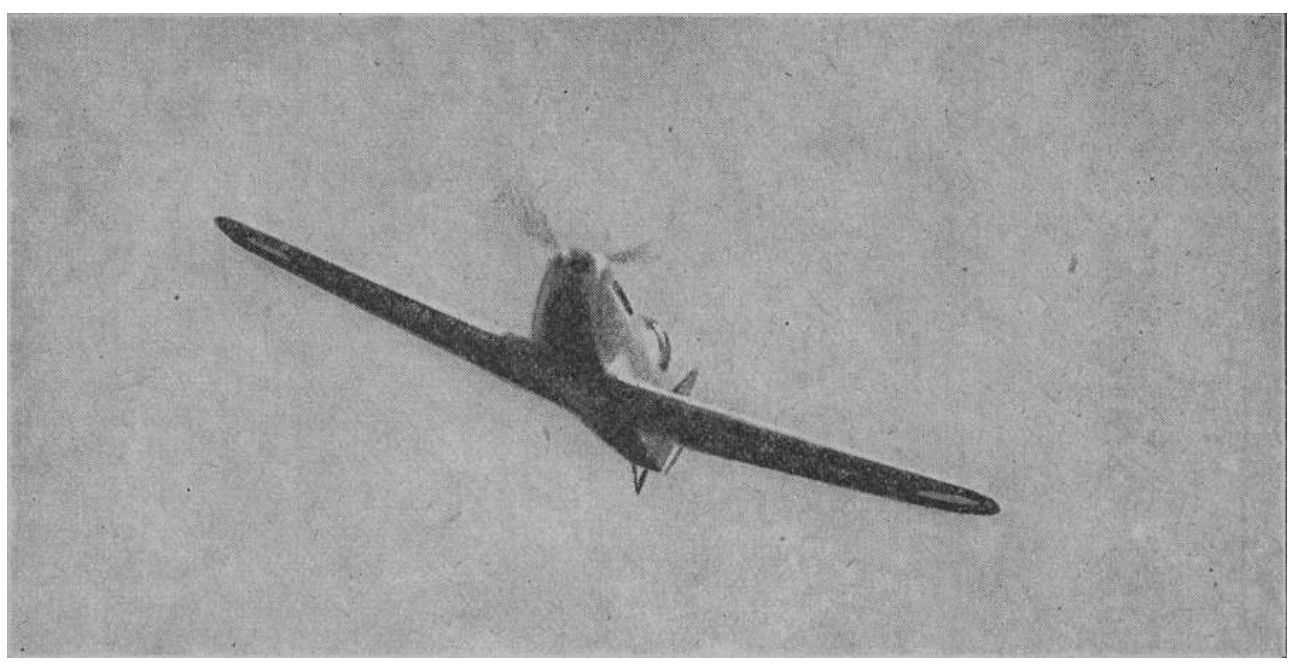

Meilleur avion de chasse français de la Seconde Guerre mondiale, capable, dans certaines conditions, de rivaliser avec les appareils allemands, en raison de ses qualités de manœuvrabilité, le Dewoitine D. 520 se distingue de son devancier, le D.510, par ses performances mais aussi sa conception qui permet d'envisager rapidement une mise en production en séries. II entre en ligne progressivement, pendant la Drôle de guerre, mais reste rare dans le ciel français, en mai et juin 1940, ne lui permettant pas, dans ces conditions, de peser réellement sur le sort des combats.

Extrait de La science et la vie, avril 1940

19 La débâcle du Bloch n'est pas passée inaperçue et le changement de paradigme dans la façon dont les ingénieurs français développaient leurs designs correspondait à ce retour d'expériences. Mais les conséquences des choix antérieurs sont encore bien présentes à la veille d'engager le combat. Le changement est trop tardif pour porter efficacement ses fruits. De la Grange, rapporteur du Budget de l'Air au Sénat, était très critique envers les ingénieurs français et inquiet de l'état de l'aéronautique française. Il se livrait à une comparaison sévère par rapport aux choix des autres pays. Dans une lettre adressée à Pierre Cot relative à la fabrication des Morane-Saulnier, il incrimine les méthodes de fabrication, conséquences des caractéristiques de la conception à la française :

«C'est un appareil que vous ne pouvez pas construire en séries importantes avec de gros outillages, car c'est un appareil conçu par des ingénieurs qui ne savent pas ce que c'est qu'une machine-outil, de sorte que si nous voulons entrer dans le fond du problème et concevoir enfin qu'avant de vouloir produire des avions comme font 
les Allemands, les Américains et maintenant les Anglais, en grandes séries, il ne suffit pas de donner de l'argent au Ministre de l'Air ${ }^{47}$, il ne suffit pas d'acheter des machines-outils, il faut encore réformer la mentalité des ingénieurs qui font nos prototypes ${ }^{48}$ ».

Fig.4. Le chasseur monoplace Morane-Saulnier MS 406

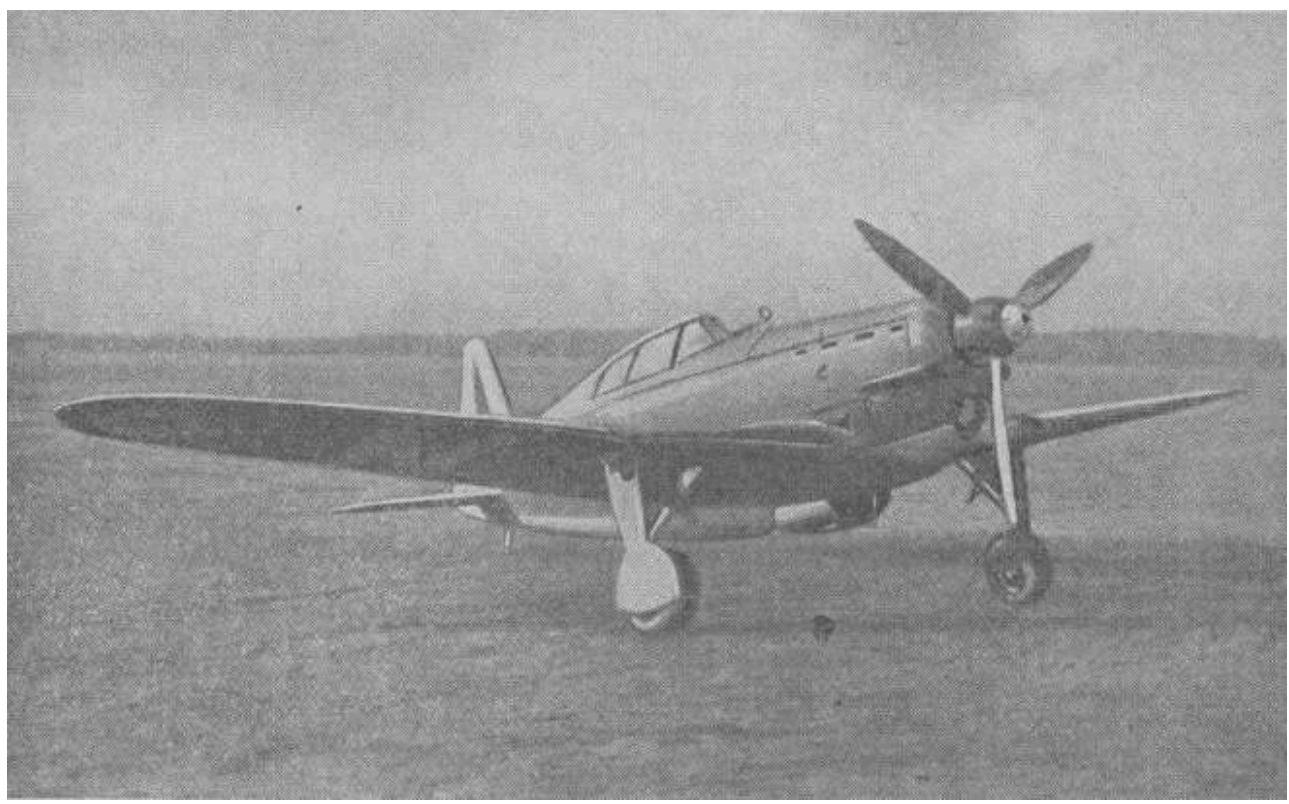

Avion de chasse le plus répandu au sein de l'Armée de l'air, au moment de la campagne de France du printemps 1940, il se distingue par sa grande maniabilité et une facilité de pilotage qui le font apprécier par les pilotes, mais aussi par son incapacité à se confronter aux meilleurs chasseurs allemands, nettement plus rapides. Sa construction repose sur un ensemble de différents matériaux rendant son assemblage complexe.

Extrait de La science et la vie, avril 1940

Selon les Britanniques :

\begin{abstract}
«Les Français ne semblaient pas avoir saisi les caractéristiques sousjacentes de la production à grande échelle, et leur principal objectif était la production d'un taux de 300 à 330 machines par mois. Il semblait évident que, aussi longtemps que les Français ne visaient qu'à un tel taux de production, ils ne l'attendraient pas ${ }^{49}$ ".
\end{abstract}

21 En raison de la complexité de la construction, du nombre de pièces complexes et des changements fréquents de design, l'application des techniques de production en masse était difficile à introduire dans l'industrie aéronautique française. La création d'une pièce aussi courante qu'une soupape exigeait d'immenses efforts de recherche et de mise en point. Chez Hispano-Suiza, 111 opérations individuelles étaient nécessaires, à partir de différentes machines et ceci pour chaque soupape ${ }^{50}$. Les moteurs HispanoSuiza en possédaient généralement $24^{51}$. Il fallait donc, pour les soupapes d'un seul moteur, 2664 opérations impliquant plusieurs machines, sans tenir compte des pièces rejetées. Les soupapes devaient supporter de grands écarts de température sans se déformer ou perdre en performance. Pendent les tests, chaque soupape devait supporter 800 degrés Celsius ${ }^{52}$. Le métal utilisé dans les soupapes devait résister aux hautes températures et aux produits d'échappement. Des alliages d'acier, de chrome, et 
de nickel ont tout d'abord été essayés, ce mélange correspondant bien aux exigences mécaniques et thermiques ${ }^{53}$. Pourtant, pour faire les forgeages de la cavité de la soupape, il fallait un métal très malléable. Celui qui répondait aux contraintes physiques ne correspondait pas aux possibilités d'usinage.

Hispano-Suiza a donc consulté des aciéristes et mené des campagnes d'essais, afin de trouver un matériau qui répondait à la fois aux caractéristiques de l'appareil mais aussi aux attentes industrielles au moment de sa mise en production. Une combinaison d'acier, de chrome et de silicium a fini par correspondre à tous ces aspects. À sa livraison à l'usine, sous forme de barres, des épreuves physico-chimiques étaient réalisées sur chaque lot. Le métal qui devait servir à produire des soupapes était traité avec de l'acide afin de trouver des défauts comme des criques, des pailles et des tapures, pouvant conduire à son éventuel rejet. Après plus d'un an d'essais pour réaliser une soupape creuse, les techniciens sont parvenus à bonnes fins en utilisant une presse de 400 tonnes qui poinçonnait les lopins d'acier, une batterie de fours et des machines rotatives destinées, par étapes successives, à former la pièce en «tonneau » et, enfin, en « gobelet ${ }^{54}$.

Fig.5. Le temps de production des avions militaires français (Morane-Saulnier 406, Dewoitine 520 Bloch 174), 1932-1939)

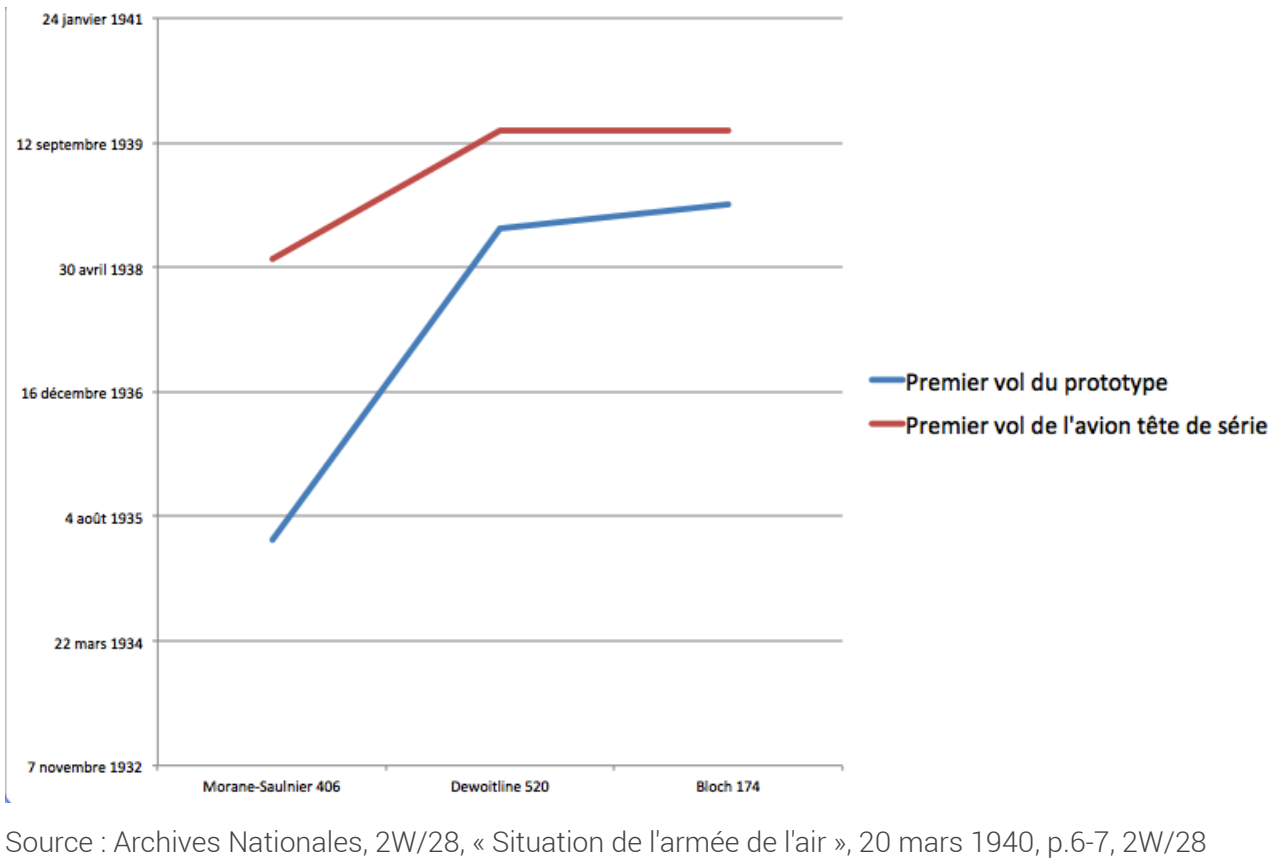

Le graphique ci-dessus fait ressortir le temps nécessaire au développement de trois avions français pendant la période du réarmement. Les améliorations pour mener un avion depuis son prototype jusqu'à la mise en production sont visibles. Elles correspondent à un apprentissage de la part de l'industrie ou, tout au moins, des ingénieurs, dans le but d'accélérer la mise en service d'un appareil. Pour le Morane, le délai était encore de trente-sept mois; pour le Dewoitine, il tombait à treize mois (ce qui correspondait à une diminution du temps nécessaire de 64,86\%) et, enfin, pour le Bloch, à seulement dix mois (ce qui représentait une réduction de temps supplémentaire de 23,07\% par rapport au Dewoitine). Les améliorations des délais 
d'industrialisation entre le Dewoitine et le Bloch d'une part et le Morane-Saulnier d'autre part provenaient de l'articulation entre la conception et la production en série.

Les conditions d'élaboration, de mise au point et d'industrialisation avaient donc été revues. Il est intéressant de noter que les spécifications auxquelles répondaient le Morane-Saulnier et le Dewoitine correspondaient respectivement aux années $1934^{55}$ et $1936^{56}$. Le Morane-Saulnier n'avait besoin que d'une année avant de prendre son envol pendant que deux avaient été nécessaires pour le Dewoitine. Au moment sa mise en production, le Morane-Saulnier 406 nécessitait de 14000 à 20000 heures ${ }^{57}$. Par contre, en raison de sa construction simple, associée à l'utilisation du soudage électrique et du rivetage, le Dewoitine 520 était mieux adapté à la production de masse que ses prédécesseurs. Il fallait 7000 heures pour le construire, en comparaison aux 14000 heures nécessaires à la fabrication de son prédécesseur, le Dewoitine 510, un avion beaucoup moins performant ${ }^{58}$. Le délai nécessaire pour concevoir un avion de qualité et réalisable en grande série avait fait ressortir une complexité que ne révélaient pas les pratiques précédentes, en priorité orientées vers la performance pure de l'appareil.

\section{Conclusion}

De sa conception à sa sortie d'usine, et même très souvent après, un avion était exposé à des changements potentiels de design et de conditions de construction. Les premiers apparaissaient comme un mal nécessaire. L'évolution d'un avion se faisait par tâtonnements, avec de petites modifications qui devaient ensuite être testées pour, éventuellement, être retenues. C'est pourquoi le développement d'un avion prenait souvent de 4 à 7 ans (Zeitlin 1995: 53). Avec une période aussi longue, un avion performant pouvait être déclassé et même obsolète au moment où il entrait en dotation du fait de l'évolution rapide de la technologie aéronautique. De plus, l'augmentation exponentielle du niveau de production pendant le réarmement a nécessité l'emploi d'autres techniques de production, pour lesquelles les avions antérieurs n'avaient pas été pensés. Les ingénieurs étaient donc habitués à proposer des avions performants, sans trop de soucier des conditions de production. À la veille de la Seconde Guerre mondiale, les ingénieurs français, tiraillés entre les demandes changeantes des militaires, le progrès technique et les exigences de la production de masse, ont vu leur réputation être ternie par le manque d'avions performants en service. Pourtant, la courbe d'apprentissage dans la navigation fait ressortir une réduction des délais, ainsi qu'une amélioration des performances à l'aube de la guerre : les retours d'expériences avaient été assimilés, mais de manière trop tardive.

\section{BIBLIOGRAPHIE}

AGNEW Kenneth, «The Spitfire: Legend or History? An Argument for a New Research Culture in Design ", Journal of Design History, vol.6, n², 1993, p.126 
COHEN Yves, « The Modernization of Production in the French Automobile Industry between the Wars: A Photographic Essay », The Business History Review, vol.65, n²4, 1991, p.772

GARRAUD Philippe, «L'action de l'armée de l'air en 1939-1940: Facteurs structurels et conjoncturels d'une défaite », Guerres mondiales et conflits contemporains, ${ }^{\circ}{ }^{2} 202 / 203$, avrilseptembre 2001

GARRAUD Philippe « Les contraintes industrielles dans la préparation de la guerre de 1939-1940 » Guerres mondiales et conflits contemporains, juillet-septembre 2002, $\mathrm{n}^{\circ} 207$

LUCAS Yvette, «L'aéronautique, une industrie productrice de savoir-faire », Le Mouvement social, n¹45, décembre 1988

SIMONSON G. R., « The Demand for Aircraft and the Aircraft Industry, 1907-1958 », The Journal of Economic History, vol.20, ${ }^{\circ} 3$, septembre 1960

ZEITLIN Jonathan, « Flexibility and Mass Production at War : Aircraft Manufacture in Britain, the United States, and Germany, 1939-1945 », Technology and Culture, vol.36, n¹, janvier 1995, p.46-79

\section{NOTES}

1. « Aero Engine Development », Flight, n 1378, vol. XXVII, 23 mai 1935, p.569.

2. MACGILL Elizabeth M. G., « Mass production: Some factors affecting its achievement », Flight, $\mathrm{n}^{\circ} 1656$, vol. XXXVIII, 19 septembre 1940, p.229.

3. De SEVERSKY Alexander, «Some ideas on the war: An American designer comments on escort fighters, the Spitfire and Messerschmitt and other matters of interest » Flight, $n^{\circ} 1643$, vol. XXXVII, 20 juin 1940, p.546.

4. «speed, maneouvrability and assurance of adequate power [...] ease of control at critical moments [...] all protection possible for the pilot [...] built within the compromise between the need for an instrument of defence of high efficiency and the need for a machine of high aerodynamic perfection ", Ibidem.

5. MILES Frederick George, «Track Assembly », Flight, n¹652, vol. XXXVIII, 22 août 1940, p.146.

6. «Control Problems Discussed », Flight, n¹564, vol. XXXIV, 15 décembre 1938, p.h.

7. « Designing for Speed », Flight, $\mathrm{n}^{\circ} 1641$, vol. XXXVII, 6 juin 1940, p.d.

8. POLLARD H. J., "An Aircraft Engineer in Paris: The 1938 Aero Show Revisisted », The Aircraft Engineer, Supplement to Flight, ${ }^{\circ} 155$, vol. XIV, n¹2, 29 décembre 1938, p.606b.

9. BUTTERS J. Keith, GILMORE Frank F., HUNT Pearson, LAWLER Paul F. et LILLEY Tom, " Problems of Accelerating Aircraft Production during World War II », Division of Research, Graduate School of Business Administration, Harvard University, 1946, p.39.

10. MACGILL Elizabeth M. G., « Mass Production... », op.cit., p.229.

11. KING Horace Frederick, "The Trend of Small-Fighter Design », Flight, n ${ }^{\circ} 1402$, vol. XXVIII, 7 novembre 1935, p.a.

12. FEDDEN Alfred Hubert Roy, « War - And the Development of the Aero-Engine », Flight, $\mathrm{n}^{\circ} 1616$, vol.XXXVI, 14 décembre 1939, p.d.

13. "Until the first production aircraft has flown away there is no certainty in design at all ", MILES F. G., «Track Assembly: Line System in Use at the Works of Phillips and Powis ", Flight, $n^{\circ} 1652$, vol. XXXVIII, 22 août 1940, p.147.

14. MACGILL, Elizabeth M. G., « Mass Production... », op.cit., p.229.

15. BUTTERS J. Keith et. al., « Problems of Accelerating Aircraft... », op.cit., p.19.

16. MACGILL Elizabeth M. G., « Mass Production... », op.cit., p.229.

17. Ibidem, p.228. 
18. Archives Nationales (AN), 2W/28, Note 3, annexe 2, le 27 janvier 1941, p.62.

19. BUTTERS J. Keith et al., « Problems of Accelerating Aircraft... ", op.cit., p.44.

20. Ibidem, p.42.

21. Ibid.

22. «The Industry: Steel Bar Specifications », Flight, n¹585, vol. XXXV, 11 mai 1939, p.488.

23. POBJOY Douglas Rudolf, «Engine Design and Materials», Flight, n 1564 , vol. XXXIV, 15 décembre 1938, p.c.

24. Ibidem, p.550.

25. THOMAS F. M., et TAWELL A. G. A., «Experiments with Composite Airscrew Blades ", The Aircraft Engineer, $\mathrm{n}^{\circ} 161$, vol. XVII, N6, 29 juin 1939, p.664a.

26. «The Outlook: Towards an Ideal », Flight, $\mathrm{N}^{\circ} 1573$, vol. XXXV, 16 février 1939, p.150.

27. THOMAS F. M., et TAWELL A. G. A., « Experiments... », op.cit., n¹61, p.664a.

28. ANDREWS W. R., "Static Thrust of Airscrews ", The Aircraft Engineer, Supplement to Flight, $\mathrm{n}^{\circ} 128$, vol. XIII, $1^{\mathrm{er}}$ octobre 1936, p.14.

29. FEDDEN Alfred Hubert Roy, « War... », op.cit., p.c.

30. MILES F. G., « Track Assembly: Line System in Use at the Works of Phillips and Powis », Flight, n652, vol. XXXVIII, 22 août 1940, p.147.

31. GREEN William, War planes of the Second World War: Fighters, Vol. 1, Garden City, New York, Hanover House, 1960, p. 31.

32. AN, 2W/28, note 3, annexe 2, le 27 janvier 1941, p.50.

33. «The Hawker Hurricane: The Birth and Growth of a Great Fighter Described » Flight, le 31 octobre $1940, \mathrm{n}^{\circ} 1662$, vol. XXXVIII), p.e.

34. AN, 2W/28, «L'établissement de l'exécution du Plan V de guerre » s.d., p.73.

35. Musée de l'Air et de l'Espace, boîte Dewoitine, Extrait des mémoires de Marcel Doret, s.d.

36. AN, $2 \mathrm{~W} / 28$, «Observations présentées au sujet du rapport de M. le contrôleur Chossat sur le Plan V », Note 13, s.d., p.31.

37. AN, 2W/28, Note 3, annexe 2, le 27 janvier 1941, p.68.

38. MACGILL, Elizabeth M. G., « Mass Production... », op.cit., p.228.

39. BUTTERS J. Keith et. al., « Problems of Accelerating Aircraft... ", op.cit., p.42.

40. Ibidem.

41. AN, $2 \mathrm{~W} / 28$, «Observations présentées au sujet du rapport de M. le contrôleur Chossat sur le Plan V », note 13, s.d., p.13.

42. CRISTESCO, Michel « The M. Bloch $151 \& 152 »$, Profile Publications, $n^{\circ} 201,1967$, p.3.

43. GREEN William, War planes..., op.cit.,1960, p.27-28.

44. "The Paris Aero Show: Heavy Predominance of Military Aircraft », Flight, n¹560, vol. XXXIV, $1^{\text {er }}$ décembre 1938, p.489.

45. Société Nationale de Constructions Aéronautiques du Midi - Dewoitine D.520 - Bureau pour la vente à l'étranger de matériel aéronautique, sd.

46. «The Paris Aero Show... », op.cit., p.491.

47. AN, $2 \mathrm{~W} / 28$, «Observations présentées au sujet du rapport de M. le contrôleur Chossat sur le Plan V », note 13, s.d., p.13.

48. Le général Gamelin, par contre, dont l'opinion contrastait fortement avec celle de Chossat, affirmait que le développement de l'armée de l'air était « avant tout une question d'argent ». AN, $2 \mathrm{~W} / 27$, note du général Gamelin au président du Conseil et au ministre de la Défense nationale et de la Guerre, « Note sur la situation actuelle 936/DN.3 », le 26 octobre 1938, p.1.

49. "The French did not seem to have grasped the underlying features of large-scale production, and their ultimate objective was production at the rate of 300-330 machines a month. It seemed clear that, so long as the French only aimed at a rate of production of this kind, they would not achieve it. », AN, «Cabinet 18 (39) », compte-rendu d'une réunion du Cabinet, le 5 avril 1939, annexe, p.1. 
50. Musée de l'Air et de l'Espace, boîte Hispano-Suiza, BALLERET A., «Les soupapes creuses refroidies au sodium », Revue technique Hispano-Suiza, n², octobre 1938, p.14.

51. Musée de l'Air et de l'Espace, boîte Hispano-Suiza, SAUVY et HÉBRARD, «Problèmes d'outillage : Les machines spéciales ", Revue technique Hispano-Suiza, juillet 1938, nº 1, p.13.

52. Musée de l'Air et de l'Espace, boîte Hispano-Suiza, BALLERET, A., «Les soupapes creuses refroidies au sodium », Revue technique Hispano-Suiza, n², octobre 1938, p.6.

53. Ibidem, p.7.

54. Ibid., p.8.

55. BOTQUIN Gaston, « The Morane Saulnier 406 », Profile Publications, n¹47, 1967, p.3.

56. DANEL Raymond, « The Dewoitine $520 »$, Profile Publications, $n^{\circ} 135,1971$, p.1.

57. Niveau qui, par la suite, fut réduit à 8000 heures par unité, « Observations présentées au sujet du rapport de M. le contrôleur Chossat sur le Plan V » AN, 2W/28, note 13, s.d., p. 13.

58. « France's Fastest Fighter », Flight, n¹633, vol.XXXVII, 11 avril 1940, p.f.

\section{RÉSUMÉS}

Concevoir et fabriquer un avion en France dans les années 1920-1930, c'était affronter deux problèmes. D'une part, les ingénieurs étaient habitués à concevoir des avions performants sans réfléchir aux conditions de production. D'autre part, il était habituel qu'un modèle reçoive des changements potentiels dans son design et dans ses conditions de fabrication, de sa conception à sa sortie d'usine, et même très souvent après. L'évolution s'en faisait par tâtonnements, avec de petites modifications que l'on testait éventuellement avant de les retenir. Au total, le processus conception/fabrication/développement ne durait pas moins de 4 à 7 ans, avec pour résultat du fait de l'évolution rapide de la technologie aéronautique, qu'un avion performant pouvait être déclassé et même obsolète au moment où il entrait en dotation. À la veille de la Seconde Guerre mondiale, tiraillés entre les demandes changeantes des militaires, le progrès technique et les exigences de la production de masse, les ingénieurs français ont vu leur réputation ternie par le manque d'avions performants disponibles. Pourtant, la courbe d'apprentissage dans la navigation montre une réduction des délais, ainsi qu'une amélioration des performances à l'aube de la guerre, signe que les retours d'expériences avaient été assimilées. C'est l'accélération de ce processus de conception/fabrication qu'étudie cet article, en s'appuyant, entre autres, sur l'analyse « en direct » qu'en faisait la presse technique anglo-saxonne de l'époque.

Designing and manufacturing aircraft in the inter-war period in France meant facing two problems. On the one hand, engineers were used to designing high-performance aircraft without thinking about production conditions. On the other hand, it was usual for a model to receive potential changes in its design and manufacturing conditions, from the time it was designed until it left the factory, and very often afterwards. In this trial-and-error design, small modifications were tested before they were possibly adopted. The whole process generally took 4 to 7 years with the result that a high-performance aircraft could be downgraded and even become obsolete by the time it was put into service, due to the rapid evolution of aeronautical technology. On the eve of the Second World War, French engineers who had to cope with the changing demands of the military, technical progress and the requirements of mass production, saw their reputation tarnished by the lack of available high-performance aircraft. Yet the learning curve in navigation shows a reduction in lead times, as well as an improvement in performance at the dawn of the 
war, a sign that the feedback had been assimilated. This article analyses this design/ manufacturing process and its acceleration by studying mainly the "live" analysis made by the Anglo-Saxon technical press of the time.

INDEX

Mots-clés : histoire des techniques, avion, armement, conception, ingénierie, aéronautique, ingénieur

Keywords : history of technology, aeronautics, airplane, aviation, design, warfare, engineering, industrial design

\section{AUTEUR}

\section{DAVID CULPEPPER}

David Culpepper a obtenu un master en histoire des techniques à l'Université Paris 1 PanthéonSorbonne, en 2013-2014, avec un mémoire intitulé La perception des avions de chasse français pendant l'entre-deux guerres et 1939-1940. Il a ensuite débuté une thèse sur l'histoire technique de l'aéronautique militaire en France de 1933 à 1940. 